\title{
Factores que dificultan la inseminación artificial en ovinos, y su impacto en las tasas de fertilidad, preñez y parto. Revisión sistemática de literatura
}

\author{
Daniela Cáceres Bautista*, EMVZ, Edgar Mauricio Mogollón W., Ph.D.
}

${ }_{1}$ Facultad de Medicina Veterinaria y Zootecnia, Universidad Cooperativa de Colombia, Sede Bucaramanga, Colombia.

Recibido: 31 de enero del 2019

Aprobado: 31 de julio del 2019

*Autor de correspondencia: Daniela Cáceres Bautista, Facultad de Medicina Veterinaria y Zootecnia, Universidad Cooperativa de Colombia, Bucaramanga, Colombia, calle 30A n. 33-51, of. 603, (57) 3188294942 ,

correo electrónico: caceresbautistadaniela@gmail.com; daniela.caceresb@campusucc.edu.co

Cómo citar este artículo: Cáceres-Bautista D, Mogollón EM. Factores que dificultan la inseminación artificial en ovinos, y su impacto en las tasas de fertilidad, preñez y parto. Revisión sistemática de literatura. Spei Domus. 2017; 13(26-27):1-13. doi: https://doi.org/10.16925/2382-4247.2017.01.05

Resumen. Introducción: la creciente importancia de la producción ovina en Colombia, su inventario nacional de 1.578 .684 , la producción en toneladas de carne/año y el incremento de su consumo per cápita de 350 a 500 gramos por persona/año, demanda intervenciones para mejorar los indicadores reproductivos de la especie, con el apoyo, entre otros, del uso de las biotecnologías reproductivas. Por esto es pertinente identificar los factores que dificultan la inseminación artificial (IA) en ovinos, con el fin de intervenir sobre ellos y contribuir a la mejora de sus indicadores reproductivos y el bienestar de los productores. Metodología: se realizó una revisión sistemática de literatura para identificar los principales factores reportados, que dificultan la preñez vía inseminación artificial en ovinos. Para la selección de artículos, se tuvo en cuenta su carácter de acceso abierto, y se consultaron productos de investigación relacionados con los parámetros morfométricos, técnicas que de IA que atraviesan el cérvix (IAV, IAC, IATC) e IA laparoscópica, formas de conservación del semen (fresco, refrigerado y congelado-descongelado) y protocolos de sincronización de celo, que incluyeran dentro de sus resultados tasas de fertilidad, preñez o partos. Se excluyeron artículos escritos en idiomas diferentes al español, portugués e inglés, especies diferentes a la ovina, lo mismo que resultados que no incorporaban protocolos de sincronización, la presentación del semen, el tipo de técnica de IA utilizada, las tasas de fertilidad, preñez o partos. Resultados: se seleccionaron 44 artículos los cuales cumplían con los criterios de inclusión, de los cuales 15 se relacionaban con los parámetros morfométricos, formas del orificio cervical y su correlación con la edad, y 29 se relacionaron con las técnicas de iA, los tipos de conservación del semen, protocolos de sincronización y su correlación con las tasas de fertilidad, preñez y partos. Conclusiones: este trabajo mostró que los principales factores que dificultan la preñez por IA en ovinos son: la edad de los animales, los parámetros morfométricos del cérvix, el tipo de técnica de IA y la presentación del semen.

Palabras clave: inseminación artificial, inseminación transcervical, parámetros morfométricos, técnicas IA, ovinos. 


\title{
Factors that hinder artificial insemination in sheep and its impact on fertility, pregnancy and parturition rates: systematic literature review
}

\begin{abstract}
Introduction: The increasing importance of sheep farming in Colombia, its national inventory, the production in tons of meat/year and the increase in per capita consumption, demand interventions to improve the reproductive indicators of the species, with support among others of the use of reproductive biotechnologies. For this reason, it is pertinent to identify the factors that hinder Artificial Insemination (AI) in sheep, in order to intervene on them and contribute to the improvement of their reproductive indicators and the welfare of the producers. Methodology: A systematic literature review was conducted to identify the main factors reported, which make pregnancy difficult through artificial insemination in sheep. For the selection of articles, its open access nature was taken into account, and research products related to morphometric parameters, AI techniques that cross the cervix (IAV, IAC, IATC) and laparoscopic AI, forms of conservation were consulted of semen (fresh, refrigerated and frozen-thawed) and estrus synchronization protocols, which include fertility, pregnancy or delivery rates within their results. We excluded articles written in languages other than Spanish, Portuguese and English, species other than sheep, as well as results that did not incorporate synchronization protocols, the presentation of semen, and the type of AI technique used, fertility rates, pregnancy or lambing. Results: Forty-four articles were selected, which met the inclusion criteria, of which 15 were related to morphometric parameters, forms of the cervical os and its correlation with age, and 29 were related to AI techniques, the type of semen conservation, synchronization protocols and their correlation with fertility, pregnancy and lambing rates. Conclusions: This work made it possible to clarify that the main factors that hinder AI pregnancy in sheep are the age of the animals, the morphometric parameters of the cervix, the type of AI technique and the presentation of the semen.
\end{abstract}

Keywords: Artificial insemination, transcervical insemination, morphometric parameters, IA techniques, sheep.

\section{Fatores que dificultam a inseminação artificial em ovinos e seu impacto nas taxas de fertilidade, prenhez e parto: revisão sistemática de literatura}

Resumo. Introdução: a crescente importância da produção ovina na Colômbia, seu inventário nacional de 1.578.684, a produção em toneladas de carne/ano e o aumento de seu consumo per capita de 350 a 500 gramas por pessoa/ano demandam intervenções para melhorar os indicadores reprodutivos da espécie, com o apoio, entre outros, do uso das biotecnologias reprodutivas. Por isso, é pertinente identificar os fatores que dificultam a inseminação artificial (IA) em ovinos, com o objetivo de intervir sobre eles e contribuir para melhorar seus indicadores reprodutivos e o bem-estar dos produtores. Metodologia: realizou-se uma revisão sistemática de literatura para identificar os principais fatores relatados que dificultam a prenhez via IA em ovinos. Para a seleção de artigos, levou-se em consideração seu caráter de acesso aberto e foram consultados produtos de pesquisa relacionados com os parâmetros morfométricos, técnicas de IA que atravessam o cérvix (IAV, IAC, IATC) e IA laparoscópica, formas de conservação do sêmen (fresco, refrigerado e congelado-descongelado) e protocolos de sincronização de cio, que incluíram dentro de sus resultados taxas de fertilidade, prenhez ou partos. Foram excluídos os artigos escritos em idiomas diferentes ao espanhol, português e inglês, espécies diferentes à ovina, bem como resultados que não incorporassem protocolos de sincronização, apresentação do sêmen, tipo de técnica de IA utilizada, taxas de fertilidade, prenhez ou partos. Resultados: foram selecionados 44 artigos que cumpriam os critérios de inclusão, dos quais 15 se relacionavam com os parâmetros morfométricos, com as formas do orifício cervical e com sua correlação com a idade, e 29 se relacionavam com as técnicas de IA, com os tipos de conservação do sêmen, com os protocolos de sincronização e com sua correlação com as taxas de fertilidade, prenhez e partos. Conclusões: este trabalho mostrou que os principais fatores que dificultam a prenhez por IA em ovinos são: a idade dos animais, os parâmetros morfométricos do cérvix, o tipo de técnica de IA e a apresentação do sêmen.

Palavras-chave: inseminação artificial, inseminação transcervical, parâmetros morfométricos, técnicas IA, ovinos. 


\section{Introducción}

La primera inseminación artificial (IA) exitosa la realizó el biólogo italiano Lazaro Spallanzani en 1784 en una perra, la cual parió tres cachorros 62 días después. En 1890, el médico veterinario francés Repiquet usó la inseminación en yeguas, lo que amplió su uso en las diversas especies de mamíferos. Walter Heape (1855-1919) informó sobre el uso de la IA en conejos, perros y caballos [1]. Años más tarde, en 1923, el científico ruso Ivanoff amplió el uso de inseminación artificial en vacas y ovejas. En 1928, Rusia utilizó la inseminación artificial en gran escala, e inseminó más de un millón de vacas y de 15 millones de ovejas [1].

En el contexto mundial, treinta y tres países han producido cerca del $85 \%$ de la carne ovina del planeta. China encabeza esta producción con un 30 \%, seguido de Unión de Repúblicas Socialistas Soviéticas, Nueva Zelanda, Australia, Reino Unido, Irán y Turquía. La producción de carne ovina en América Latina es liderada por Brasil con 8.000 toneladas, México con 53.740, Argentina con 46.500 y Colombia participa con 7.503 toneladas/ año [2].

Según el ICA, en el 2017 los departamentos con mayor población ovina son la Guajira con 641.897 cabezas, seguido del Magdalena con 107.441, Boyacá con 105.937, Cesar con 101.177, y Córdoba con 96.653. El departamento de Santander, a pesar de que no encabeza la lista, culturalmente es uno de los mayores consumidores de carne ovina, lo cual se ve reflejado en la participación total de todos sus municipios, en donde produce 55.482 cabezas ovinas [3], [4].

En consideración al inventario nacional de ovinos en Colombia, las toneladas de carne/año y el consumo per cápita, con el interés de los productores por el uso de las biotecnologías reproductivas, se realiza esta investigación, para identificar los principales factores que dificultan la IA en ovinos, con el fin de intervenirlos y facilitar e incrementar su uso por parte de los productores [2].

\section{Materiales y métodos}

Identificación y recolección de la información.

Se realizaron las búsquedas de literatura en Pubmed, Scopus, Proquest, Sciencedirec y Scielo de artículos sobre los parámetros morfométricos del cérvix ovino, técnicas de inseminación artificial, protocolos se sincronización y técnicas de conservación del semen realizados con los siguientes términos (Sheep AND artificial insemination AND ovines) OR ("ewes AND artificial insemination OR transcervical") OR ("semen AND artificial insemination")

\section{Criterios de inclusión}

Se realizó una revisión sistemática de literatura de los artículos relacionados, se unificaron las edades de los animales evaluados en los artículos, por medio de la cronometría dentaria de dicha especie, estableciendo que animales $<12$ meses son animales jovenes, ya que aún cuentan con sus dientes de leche $\mathrm{y}$ animales $>12$ meses que presente al menos un par de dientes permanentes se considera animales adultos.

Se revisaron artículos científicos relacionados con los tipos de ia que atraviesan el cérvix, IA laparoscópica, tipos de conservación del semen (fresco, refrigerado y congelado-descongelado, tipos de sincronización de celo, que dentro de sus resultados determinen tasas de fertilidad, preñez y partos.

Se establecieron dos protocolos de sincronización con relación al tiempo de permanencia del dispositivo intravaginal: corto: $<9$ días y largo $>9$ días.

No fueron considerados idiomas diferentes al español, inglés y portugués, y especies diferentes al ovino. Tampoco fueron tenidos en cuenta artículos que no incluían el protocolo de sincronización, el tipo de técnica ia, la presentación del semen, o aquellos que no mostraran de manera explícita las tasas de fertilidad, preñez o partos.

\section{Resultados y discusión}

Al realizar la búsqueda de literatura general sobre artículos científicos relacionados con los tipos de inseminación artificial (cervical, transcervical e intrauterina por laparoscopia), tipo de semen usado: fresco, refrigerado y congelado; tipos de sincronización (largo y corto), se encontraron cuarenta y cinco artículos a nivel mundial (figura 1).

En la revisión sistemática se encontraron artículos a nivel mundial de países como India, Australia, Irán, Japón, España, Tailandia, Reino Unido, Irlanda, China, Portugal, Noruega, Turquia 
y otros artículos a nivel de América Latina. En lo referente a los artículos sobre inseminacion artificial en ovinos, se pudo evidenciar que el país de América que más desarrollo investigativo tiene en esta area es Brasil seguido por Perú, Canadá y Uruguay.

\section{La edad y su relación con los valores morfometricos}

Se creería que un cérvix corto representa menor complejidad a la hora de realizar IA, debido a la menor distancia que existe entre cada anillo cervical, permitiendo así una mayor penetración del catéter; pero según M. Kaabi et al. [5], M. Souza et al. [6], C. A. Cruz Junior et al. [7] y M. C. Franco et al [8] un cérvix corto tiende a hacer estrecho y a presentar mayor número de anillos cervicales, así como lo podemos observar en los valores explícitos en la tabla 1, por lo cual representa mayor complejidad en el proceso de inseminación artificial, debido a la interdigitación, la excentricidad de los anillos y las distancias cortas entre los anillos, que limitan el paso del catéter. Aunque en el estudio realizado por Gültiken et al. [9] se determina que el cérvix corto representa una ventaja en la raza karayaca, pues esas distancias cortas entre anillos, permiten mayor profundidad en la penetración. M. Kaabi et al. [5] describieron un cérvix largo, ancho y con un menor número de anillos cervicales, que tiene menor dificultad para el paso del catéter durante la IA. Entonces un cérvix corto, estrecho y con un número mayor de anillos cervicales, ofrece mayor dificultad para la IA. Esta característica ocurre principalmente en hembras jóvenes, al no sufrir previamente los cambios morfológicos asociados a la gestación y al parto. Autores como: M. Kaabi et al. [5], S. M. K. Naqvi et al. [11], N. Gültiken et al. y J. Eppleston el al. [12], [13] expresan que los valores morfométricos varían con la edad de los animales. Las ovejas jóvenes tienen su cérvix corto, cónico y sus anillos están ligeramente alineados en comparación con el cérvix de las ovejas adultas, con su cérvix más alargado, asimétrico y con menor número de anillos cervicales, circunstancia que facilita la penetración cervical [11], [13]. Según M. Kaabi et al. [5] y R. B. DUN [15] estas variaciones morfométricas se deben a la preñez y al efecto del parto sobre la estructura cervical, los cuales promueven su expansión, y probablemente la ruptura de la mayoría de los anillos cervicales.

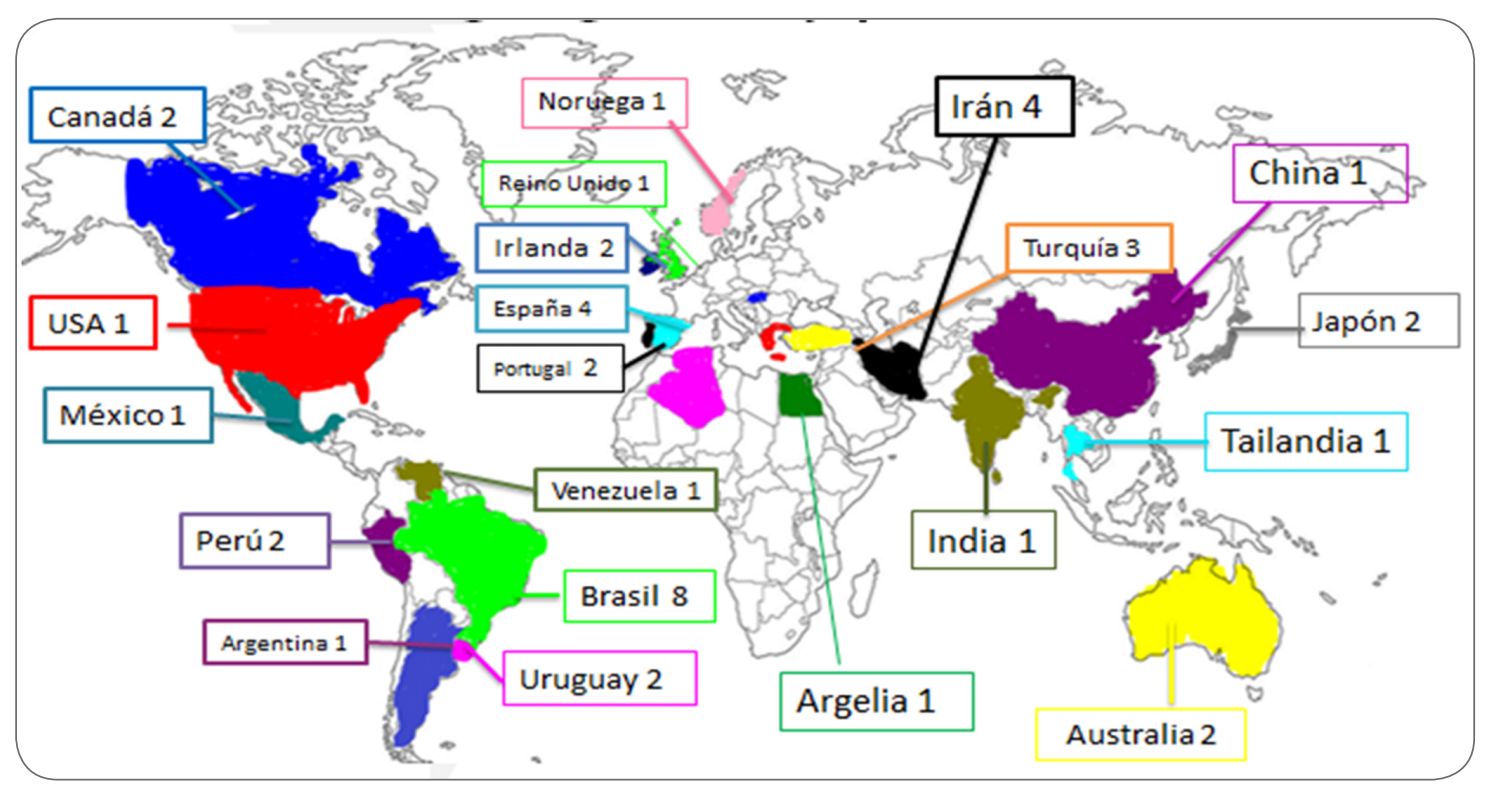

Figura 1. Mapa de la ubicación geográfica de los artículos analizados Fuente: elaboración propia 
Tabla 1. Morfometría cervical y su relación con la edad

\begin{tabular}{|c|c|c|c|c|c|}
\hline Autor & Raza & $\begin{array}{c}\text { Edad }<12 \text { meses } \\
\text { ovejas }>12 \text { meses } \\
\text { ovejas adultas }\end{array}$ & $\begin{array}{l}\text { Longitud cervical } \\
\qquad(\mathrm{cm})\end{array}$ & $\begin{array}{l}\text { Diámetro } \\
\qquad(\mathrm{cm})\end{array}$ & $\begin{array}{l}\text { Número de } \\
\text { pliegues }\end{array}$ \\
\hline \multirow{4}{*}{ M. Kaabi et al., 2006 [5] } & Merino & \multirow{4}{*}{ Ovejas adultas } & $7,09 \pm 0,13$ & $1,44 \pm 0,03^{*}$ & $3,84 \pm 0,11$ \\
\hline & Castellana & & $6,89 \pm 0,15$ & $1,37 \pm 0,04^{*}$ & $3,97 \pm 0,14$ \\
\hline & Assaf & & $7,38 \pm 0,16$ & $1,31 \pm 0,04^{*}$ & $4,63 \pm 0,14$ \\
\hline & Churra & & $6,17 \pm 0,15$ & $1,25 \pm 0,04^{*}$ & $4,54 \pm 0,13$ \\
\hline \multirow{2}{*}{ Souza MIL, et al., 1994 [6] } & Corriedale & \multirow{2}{*}{ Ovejas adultas } & $5,9 \pm 0,9$ & \multirow{2}{*}{$0,8 \pm 0,1 \mathrm{DI}$} & $4,6 \pm 0,7$ \\
\hline & Ideal & & $5,7 \pm 0,8$ & & $4,9 \pm 1,1$ \\
\hline \multirow{2}{*}{ N. Gültiken et al., 2009 [9] } & \multirow{2}{*}{ Karayaca } & Ovejas jóvenes & $2,89 \pm 0,35$ & $0,68 \mathrm{DI}^{* *}$ & $5,16 \pm 0,93$ \\
\hline & & Ovejas adultas & $3,69 \pm 0,65$ & $0,78 \mathrm{DI}^{\star *}$ & $4,73 \pm 0,7$ \\
\hline \multirow{2}{*}{ S.M.K. Naqvi et al., 2003 [11) } & \multirow{2}{*}{ Malpura Kheri } & Ovejas jóvenes & $3,87 \pm 0,12$ & $0,55 \mathrm{DI}^{* * *}$ & $3,24 \pm 0,19$ \\
\hline & & Ovejas adultas & $5,36 \pm 0,15$ & $0,65 \mathrm{DI}^{* * *}$ & $3,48 \pm 0,20$ \\
\hline \multirow{2}{*}{ J. Eppleston, 1994 [12] } & \multirow{2}{*}{ Merino } & Ovejas jóvenes & $3,69 \pm 0,11$ & $0,53 \pm 0,02 \mathrm{DI}$ & $4,21 \pm 0,13$ \\
\hline & & Ovejas adultas & $4,87 \pm 0,10$ & $0,63 \pm 0,02 \mathrm{DI}$ & $3,97 \pm 0,13$ \\
\hline C. A. Cruz Junior, et al., 2014 [7] & Santa Inés & Ovejas adultas & 4,59 & $0,29 \pm 0,12 \mathrm{DI}$ & 5,68 \\
\hline J. C. Breda et al., 2007 [10] & $\begin{array}{l}\text { Hampshire Down/ } \\
\text { Ile de France }\end{array}$ & Ovejas adultas & $7,2 \pm 1,1$ & $1,5^{* * * *}$ & $6,0 \pm 1,1$ \\
\hline M. C. Franco et al., 2014 [8] & Santa Ines & Ovejas adultas & $4,13 \pm 1,6$ & & $4,70 \pm 1,05$ \\
\hline \multirow{7}{*}{ G.W. Halbert., et al. 1990 [14] } & Suffolk & \multirow{7}{*}{ Ovejas adultas } & $6,6 \pm 1,1$ & - & $4,8 \pm 1,0$ \\
\hline & Cheviot & & $7,3 \pm 0,6$ & - & $5,6 \pm 0,5$ \\
\hline & Suffolk Cross & & $7,0 \pm 1,2$ & - & $4,7 \pm 0,8$ \\
\hline & Dorset & & $6,7 \pm 0,8$ & - & $4,6 \pm 0,9$ \\
\hline & Leicester & & $7,1 \pm 1,1$ & - & $4,8 \pm 1,0$ \\
\hline & Clun Forest & & $6,3 \pm 0,1$ & - & $4 \pm 0,0$ \\
\hline & Hampshire Cross & & 4,7 & - & 5 \\
\hline
\end{tabular}

Diámetro interno (ID)

${ }^{\star}$ Diámetro externo

${ }^{* *}$ Diámetro interno (se sacó el promedio del diámetro de los anillos cervicales)

***Diámetro de los anillos 1 y 2

${ }^{* * * *}$ Valor promedio del diámetro externo (se sumaron valor craneal, medial y caudal)

_valores no evaluados en los artículos

Fuente: elaboración propia

\section{El orificio externo cervical}

El orificio externo del cérvix fue descrito y dibujado por R. B. Dun [15], quien señaló que el prolapso del primer anillo cervical obstruye parcial o completamente la entrada al cérvix, dificulta la visualización del orificio, la estrada del catéter y, consecuentemente, la ejecución de la IA. G. W. Halbert et al. [14] C. M. Kershaw et al. [18] describieron las seis formas del orificio externo del cérvix.
1. El pico de pato: dos pliegues opuestos de tejido cervical que sobresale dentro de la vagina con una hendidura horizontal central como el orificio externo.

2. La hendidura: no hay salientes en la vagina anterior con una abertura similar a la abertura en el orificio del cuello uterino que da acceso al canal cervical.

3. La rosa: un grupo de pliegues cervicales que sobresalen en la vagina anterior y ocultan el orificio externo. 
4. La papila: una papila que sobresale en la vagina anterior con el os externo en su vértice.

5. El colgajo: un pliegue del tejido cervical que sobresale en la vagina anterior y cubre total o parcialmente el os externo creando la apariencia de un colgajo.

6. Espiral: los pliegues cervicales forman una espiral que sobresale en la vagina.

En la tabla 2, las formas del orificio externo cervical que se presentan con mayor porcentaje, es el colgajo en un $31,13 \%$ seguido de pico de pato con $29,06 \%$, roseta $20,54 \%$, hendidura $20 \%$, papila $11,61 \%$ y por último la forma de espiral con 2,8 \%.

A diferencia de lo expresado por R. B. Dun [15], G. W. Halbert et al. [14] concluyen que es posible el paso del catéter transcervicalmente, independientemente de la forma del orificio externo del cérvix, aunque la presencia de espacios ciegos a partir de los pliegues del tejido vaginal dificulta la identificación de la abertura del cérvix. Para S. M. K. Naqvi et al. [11], las formas del orificio cervical no tienen relación con su longitud u otras medidas del canal cervical, por lo cual no se puede usar como referencia para predecir la dificultad en la IA (cervical y transcervical). Aunque para D. Moura et al. [16], existe mayor dificultad cuando el orificio externo cervical es del tipo liso. Y para L. Richardson et al. [17], una forma compleja del orificio cervical a menudo conlleva una mayor manipulación del catéter por parte del inseminador, lo que aumenta el tiempo requerido para la inseminación y puede crear un riesgo potencial de daño tisular y mayor estrés. Para los autores M. C. Franco et al. [8], C. A. Cruz Junior et al. [7] y S. M. K. Naqvi et al. [11], la complejidad de la forma del orificio cervical difiere con la edad de los animales, y se presenta la forma de colgajo comúnmente en ovejas adultas, probablemente esta variabilidad esté relacionada por factores genéticos o el número de parto [5], [18].

\section{Consideraciones respecto a la técnica de inseminación artificial}

La revisión sistemática encontró una diversidad de factores de interés en torno a los diferentes resultados relacionados con aspectos propios de las técnicas de inseminación artificial, tales como aumento en la tasa de fertilidad, preñez y partos cuando se implementa IATC e IAL, junto a protocolos de sincronización largos y semen fresco; así como lo podemos analizar en las tablas 4, 5 y 6 .

Cuando se utilizó inseminación artificial vaginal junto con semen fresco y protocolos de sincronización largo se obtuvieron tasas de preñez y partos del $62 \%$ y del $56 \%$ [25], aunque la conservación del semen fresco y el transporte ha limitado su uso por parte de los productores [20]. A su vez, autores como L. Richardson et al. [17], y Y. Fang et al. [21] reportan tasas de preñez cercanas al $30 \%$ con semen congelado, aunque J. P. Barbas et al. [22] reportan una menor tasa de preñez del $4,4 \%$. La tasa de preñez con la técnica de inseminación artificial cervical y semen congelado, según L F. Pfeifer et al. [23], puede alcanzar valores hasta del $71,1 \%$, pero siempre y cuando se implemente celo natura, aunque J. Manes et al. [27] obtuvieron una tasa de preñez del 55,4 \% con semen fresco y uso de celo natural. Sin embargo, cuando se utiliza protocolo de sincronización largo y semen fresco autores como A. Allaoui et al. [29], Cardoso et al. [30], C. Ghalsasi et al. [34], S. Fierro, y OliveraMuzante [35] reportan tasas de preñez cercanas al $64 \%$ y cuando se utiliza el mismo protocolo de sincronización pero con semen congelado, la tasa de preñez es cercana al $34 \%$, cifra similar a la obtenida con la técnica IAV, no obstante, autores como Horta et al. [26] y M. Álvarez et al. [31] indican una tasa de preñez cercana a un $48 \%$ utilizando semen refrigerado. 
Tabla 2. Presentación de la forma de la abertura cervical según la edad y su correlación con la dificultad en la IA

\begin{tabular}{|c|c|c|c|c|c|c|c|c|}
\hline \multicolumn{9}{|c|}{ Forma de la abertura cervical } \\
\hline \multirow[b]{2}{*}{ Autor } & \multirow[b]{2}{*}{ Raza } & \multirow{2}{*}{$\begin{array}{c}\text { Edad }<12 \text { meses } \\
\text { ovejas }>12 \text { mese } \\
\text { ovejas adultas }\end{array}$} & \multicolumn{6}{|c|}{ forma de la abertura cervical } \\
\hline & & & $\begin{array}{c}\text { Pico de pato } \\
29,06 \%\end{array}$ & $\begin{array}{c}\text { Roseta } \\
20,54 \%\end{array}$ & $\begin{array}{l}\text { Colgajo } \\
31,13 \%\end{array}$ & $\begin{array}{c}\text { Papila } \\
11,61 \%\end{array}$ & $\begin{array}{l}\text { Hendidura } \\
\text { o lisa } 20 \%\end{array}$ & $\begin{array}{c}\text { Espiral } \\
2,8 \%\end{array}$ \\
\hline \multirow{2}{*}{ M. Souza et al., 1994} & Corriedale & \multirow{2}{*}{ Ovejas adultas } & 24,6 & 18,8 & 54,4 & - & - & 2,2 \\
\hline & Ideal & & 21,7 & 26,1 & 50 & - & _- & 2,2 \\
\hline \multirow{7}{*}{$\begin{array}{l}\text { G.W. Halbert et al., } \\
1990\end{array}$} & Suffolk* & \multirow{7}{*}{ Ovejas adultas } & 17 & 34 & 45 & - & - & 4 \\
\hline & Cheviot & & 25 & 75 & 0 & - & - & 0 \\
\hline & $\begin{array}{l}\text { Suffolk } \\
\text { Cross }\end{array}$ & & 0 & 50 & 38 & - & - & 12 \\
\hline & Dorset & & 20 & 40 & 20 & - & - & 20 \\
\hline & Leicester & & 0 & 50 & 20 & - & - & 0 \\
\hline & Clun Forest & & 100 & 0 & 0 & _- & - & 0 \\
\hline & $\begin{array}{l}\text { Hampshire } \\
\text { Cross }\end{array}$ & & 0 & 0 & 100 & - & - & 0 \\
\hline $\begin{array}{l}\text { M. C. Franco et al., } \\
2014 .\end{array}$ & Santa Inés & Ovejas adultas & 46 & 23 & 17 & - & - & - \\
\hline $\begin{array}{l}\text { S.M.K. Naqvi et al., } \\
2003\end{array}$ & $\begin{array}{l}\text { Malpura y } \\
\text { Kheri }\end{array}$ & Ovejas adultas & 30 & 10 & 26 & - & - & - \\
\hline $\begin{array}{l}\text { C. A. Cruz Junior et al., } \\
2014\end{array}$ & Santa Inés & Ovejas adultas & 52,5 & 16,25 & 7,5 & 8,75 & 15 & - \\
\hline D. Moura et al. 2011 & - & Ovejas adultas & 14,8 & 17,3 & 13,16 & 13,6 & 38,3 & - \\
\hline
\end{tabular}

*Solo se tomó en cuenta la raza Suffolk por la cantidad de ejemplares utilizados en el ensayo. _valores no evaluados en los artículos

Fuente: elaboración propia

Tabla 3. Diámetro de cada anillo cervical ( $\mathrm{mm})$

\begin{tabular}{|c|c|c|c|c|}
\hline Autor & Raza & Edad & Anillos & Diámetro $(\mathrm{mm})$ \\
\hline \multirow{5}{*}{ M. Álvarez et al 2001} & \multirow{5}{*}{ Churra } & \multirow{5}{*}{ Ovejas adultas } & Anillo 1 & $3,68 \pm 1,84$ \\
\hline & & & Anillo 2 & $1,04 \pm 0,50$ \\
\hline & & & Anillo 3 & $2,21 \pm 1,59$ \\
\hline & & & Anillo 4 & $1 ; 96 \pm 0,92$ \\
\hline & & & Anillo 5 & $1,84 \pm 0,91$ \\
\hline \multirow{6}{*}{ M. C. Franco et al., 2014.} & \multirow{6}{*}{ Santa Inés } & \multirow{6}{*}{ Ovejas adultas } & Anillo 1 & $9,60 \pm 2,29$ \\
\hline & & & Anillo 2 & $9,29 \pm 2,03$ \\
\hline & & & Anillo 3 & $8,59 \pm 2,00$ \\
\hline & & & Anillo 4 & $8,01 \pm 1,82$ \\
\hline & & & Anillo 5 & $7,76 \pm 1,89$ \\
\hline & & & Anillo 6 & $7,27 \pm 1,96$ \\
\hline
\end{tabular}


(viene)

\begin{tabular}{|c|c|c|c|c|}
\hline Autor & Raza & Edad & Anillos & Diámetro $(\mathrm{mm})$ \\
\hline \multirow{8}{*}{ C. A., Cruz Junior et al., 2014} & \multirow{8}{*}{ Santa Inés } & \multirow{8}{*}{ Ovejas adulta } & Anillo 1 & $3,54 \pm 1,62$ \\
\hline & & & Anillo 2 & $2,85 \pm 1,26$ \\
\hline & & & Anillo 3 & $2,77 \pm 1,01$ \\
\hline & & & Anillo 4 & $2,72 \pm 1,02$ \\
\hline & & & Anillo 5 & $2,90 \pm 1,02$ \\
\hline & & & Anillo 6 & $2,91 \pm 1,00$ \\
\hline & & & Anillo 7 & $3,20 \pm 0,84$ \\
\hline & & & Anillo 8 & $3,77 \pm 1,08$ \\
\hline \multirow{10}{*}{ N. Gültiken et al. 2009} & \multirow{10}{*}{ Karayaca } & \multirow{5}{*}{ Ovejas jóvenes } & Anillo 1 & $7,34 \pm 0,76$ \\
\hline & & & Anillo 2 & $7,43 \pm 0,82$ \\
\hline & & & Anillo 3 & $6,74 \pm 1,21$ \\
\hline & & & Anillo 4 & $6,65 \pm 0,98$ \\
\hline & & & Anillo 5 & $5,87 \pm 1,10$ \\
\hline & & \multirow{5}{*}{ Ovejas adultas } & Anillo 1 & $8,31 \pm 2,35$ \\
\hline & & & Anillo 2 & $8,28 \pm 1,42$ \\
\hline & & & Anillo 3 & $8,20 \pm 2,01$ \\
\hline & & & Anillo 4 & $7,29 \pm 1,56$ \\
\hline & & & Anillo 5 & $7,01 \pm 1,06$ \\
\hline
\end{tabular}

Fuente: elaboración propia.

Tabla 4. Técnicas de inseminación artificial vs protocolo de sincronización largo

\begin{tabular}{|c|c|c|c|c|c|c|c|c|c|c|c|c|c|c|c|c|}
\hline \multirow{3}{*}{ Autores } & \multicolumn{16}{|c|}{ PROTOCOLO DE SINCRONIZACIÓN LARGO } \\
\hline & \multirow{2}{*}{$\begin{array}{c}\begin{array}{c}\text { Técnicas } \\
\text { de IA }\end{array} \\
\text { SEMEN }\end{array}$} & \multicolumn{3}{|c|}{ IAV } & \multicolumn{3}{|c|}{ IAC } & \multicolumn{3}{|c|}{ IATC } & \multicolumn{3}{|c|}{ IAL } & \multicolumn{3}{|c|}{ Monta natural } \\
\hline & & $\mathrm{TF}$ & $\mathrm{TP}$ & $\begin{array}{c}\mathrm{T} \\
\text { Partos }\end{array}$ & $\mathrm{TF}$ & $\mathrm{TP}$ & $\begin{array}{c}\mathrm{T} \\
\text { Partos }\end{array}$ & $\mathrm{TF}$ & TP & $\begin{array}{c}\mathrm{T} \\
\text { Partos }\end{array}$ & $\mathrm{TF}$ & $\mathrm{TP}$ & $\begin{array}{c}\mathrm{T} \\
\text { Partos }\end{array}$ & $\mathrm{TF}$ & $\mathrm{TP}$ & $\begin{array}{c}\mathrm{T} \\
\text { Partos }\end{array}$ \\
\hline $\begin{array}{l}\text { R Masoudi et } \\
\text { al., 2017(25) }\end{array}$ & Fresco & - & 62 & 56 & - & - & - & - & 66 & 58 & - & 64 & 60 & - & - & - \\
\hline \multirow{2}{*}{$\begin{array}{l}\text { Anel et al., } \\
2005(24)\end{array}$} & Congelado & - & - & - & - & - & - & - & - & - & 44,89 & - & - & - & - & - \\
\hline & Refrigerado & 31,25 & - & - & - & - & - & - & - & - & - & - & - & - & - & - \\
\hline $\begin{array}{l}\text { L Richardson } \\
\text { et al, 2012(17) }\end{array}$ & Congelado & - & 27,6 & - & - & 36,2 & - & - & - & - & - & - & - & - & - & - \\
\hline \multirow{2}{*}{$\begin{array}{l}\text { J Barbas et al., } \\
2013(22)\end{array}$} & Congelado & - & - & 40 & - & - & 43,2 & - & - & - & - & - & - & - & - & - \\
\hline & Refrigerado & - & - & 4,4 & - & - & 30,2 & - & - & - & - & - & - & - & - & - \\
\hline $\begin{array}{l}\text { Y. Fang et al., } \\
2018(21)\end{array}$ & Congelado & - & 30 & - & - & 33,3 & - & - & - & - & - & - & - & - & - & - \\
\hline $\begin{array}{l}\text { Horta et al., } \\
2010(26)\end{array}$ & Refrigerado & 30,4 & - & - & 57,9 & - & - & - & - & - & - & - & - & - & - & - \\
\hline $\begin{array}{l}\text { J. Manes et } \\
\text { al., 2014(27) }\end{array}$ & Fresco & - & - & - & - & 38 & - & - & - & - & - & - & - & - & - & - \\
\hline \multirow{2}{*}{$\begin{array}{l}\text { M. C. } \\
\text { Gündüz et al., } \\
2010(28)\end{array}$} & $\begin{array}{l}\text { Monta } \\
\text { Natural }\end{array}$ & - & - & - & - & - & - & - & - & - & - & - & - & - & - & 86 \\
\hline & Congelado & - & - & - & - & - & $33,3^{*}$ & - & - & - & - & - & - & - & - & - \\
\hline
\end{tabular}


(viene)

\begin{tabular}{|c|c|c|c|c|c|c|c|c|c|c|c|c|c|c|c|c|}
\hline \multirow{3}{*}{ Autores } & \multicolumn{16}{|c|}{ PROTOCOLO DE SINCRONIZACIÓN LARGO } \\
\hline & \multirow{2}{*}{$\begin{array}{c}\begin{array}{c}\text { Técnicas } \\
\text { de IA }\end{array} \\
\text { SEMEN }\end{array}$} & \multicolumn{3}{|c|}{ IAV } & \multicolumn{3}{|c|}{ IAC } & \multicolumn{3}{|c|}{ IATC } & \multicolumn{3}{|c|}{ IAL } & \multicolumn{3}{|c|}{ Monta natural } \\
\hline & & $\mathrm{TF}$ & $\mathrm{TP}$ & $\begin{array}{c}\mathrm{T} \\
\text { Partos }\end{array}$ & $\mathrm{TF}$ & $\mathrm{TP}$ & $\begin{array}{c}\mathrm{T} \\
\text { Partos }\end{array}$ & $\mathrm{TF}$ & $\mathrm{TP}$ & $\begin{array}{c}\mathrm{T} \\
\text { Partos }\end{array}$ & $\mathrm{TF}$ & $\mathrm{TP}$ & $\begin{array}{c}\mathrm{T} \\
\text { Partos }\end{array}$ & $\mathrm{TF}$ & $\mathrm{TP}$ & $\begin{array}{c}\mathrm{T} \\
\text { Partos }\end{array}$ \\
\hline \multirow{2}{*}{$\begin{array}{l}\text { A. Allaoui } \text { et } \\
\text { al., 2014(29) }\end{array}$} & $\begin{array}{l}\text { Monta } \\
\text { Natural }\end{array}$ & - & - & - & - & - & - & - & - & - & - & - & - & - & 86,7 & - \\
\hline & Fresco & - & - & - & 64 & - & - & - & - & - & - & - & - & - & - & - \\
\hline \multirow{2}{*}{$\begin{array}{l}\text { Cardoso et } \\
\text { al., 2009(30) }\end{array}$} & Fresco & - & - & - & - & 58,8 & - & - & - & - & - & - & - & - & - & - \\
\hline & Congelado & - & - & - & - & 14,7 & - & - & - & - & - & 61,7 & - & - & - & - \\
\hline $\begin{array}{l}\text { M. Álvarez et } \\
\text { al., 2012(31) }\end{array}$ & Refrigerado & - & - & - & $38,2^{\star *}$ & - & - & - & - & - & - & - & - & - & - & - \\
\hline $\begin{array}{l}\text { K. Andersen, } \\
1973(33)\end{array}$ & Congelado & - & - & - & - & - & 29 & - & - & 54 & - & - & - & - & - & - \\
\hline \multirow{2}{*}{$\begin{array}{l}\text { P. M. Ghals- } \\
\text { asi, 1996(34) }\end{array}$} & $\begin{array}{c}\text { Monta } \\
\text { Natural }\end{array}$ & - & - & - & - & - & - & - & - & - & - & - & - & - & 83 & 72 \\
\hline & Fresco & - & - & - & - & 69 & 57 & - & - & - & - & - & - & - & - & - \\
\hline $\begin{array}{l}\text { S. Fierro, } \\
2017(35)\end{array}$ & Fresco & - & - & - & - & $66,2^{* * *}$ & - & - & - & - & - & - & - & - & - & - \\
\hline \multirow{2}{*}{$\begin{array}{l}\text { M. Rashidi et } \\
\text { al., 2013(36) }\end{array}$} & $\begin{array}{c}\text { Monta } \\
\text { Natural }\end{array}$ & - & - & - & - & - & - & - & - & - & - & - & - & 44,8 & - & - \\
\hline & Congelado & - & - & - & - & - & - & 68,1 & - & - & - & - & - & - & - & - \\
\hline $\begin{array}{l}\text { P. Naim et al., } \\
2009(37)\end{array}$ & Fresco & - & - & - & - & - & - & - & 59 & - & - & - & - & - & - & - \\
\hline $\begin{array}{l}\text { H Kohram et } \\
\text { al., 2017(39) }\end{array}$ & Fresco & - & - & - & - & - & - & - & 40 & 45 & - & - & - & - & - & - \\
\hline $\begin{array}{l}\text { R Masoudi et } \\
\text { al., 2017(40) }\end{array}$ & Fresco & - & - & - & - & - & - & - & 50 & 45 & - & - & - & - & - & - \\
\hline $\begin{array}{l}\text { L Avenda- } \\
\text { no-Reyes et } \\
\text { al., 2007(41) }\end{array}$ & Congelado & - & - & - & - & - & - & - & - & - & $56,6^{* * *}$ & - & - & - & - & - \\
\hline $\begin{array}{l}\text { FUKUI et al., } \\
2008(43)\end{array}$ & Congelado & - & - & - & - & - & - & - & - & - & - & 60 & - & - & - & - \\
\hline $\begin{array}{l}\text { E Mellisho et } \\
\text { al., 2006(44) }\end{array}$ & Congelado & - & - & - & - & - & - & - & - & - & - & 68,4 & - & - & - & - \\
\hline $\begin{array}{l}\text { G. Hidalgo et } \\
\text { al., 2015(46) }\end{array}$ & Congelado & - & - & - & - & - & - & - & - & - & - & 60 & - & - & - & - \\
\hline \multirow{2}{*}{$\begin{array}{l}\text { O Azawi, } \\
2011(47)\end{array}$} & Fresco & - & - & - & - & - & - & - & - & - & 91,5 & - & - & - & - & - \\
\hline & $\begin{array}{c}\text { Monta } \\
\text { Natural }\end{array}$ & - & - & - & - & - & - & - & - & - & - & - & - & 44,8 & - & - \\
\hline
\end{tabular}

Fuente: elaboración propia.

${ }^{*}$ se unieron las diferentes profundidades de IAC

${ }^{*}$ Se unieron los resultados de los 3 tipos de catéter

${ }^{* * *}$ Solo se tomó el protocolo con dispositivo intravaginal

$* * * *$ Se unieron las tasas de fertilidad de las tres razas

_valores no evaluados en los artículos

TF: tasa de fertilidad

TP: tasa de preñez 
A diferencia de las técnicas anteriores, al realizar la IA transcervical junto con protocolos cortos de sincronización y semen congelado, se obtuvo una mejor tasa de preñez del 46,6\% [38]. Pero cuando se utiliza un protocolo de sincronización largo y semen congelado las tasas de fertilidad y partos alcanzan un $68,1 \%$ y $54 \%$, respectivamente [33], [36], es decir, que se mejoran significativamente los parámetros reproductivos. Al implementar inseminación artificial por laparoscopia se alcanzan tasas de preñez y partos cercanas a un 63,3\% independientemente del protocolo de sincronización y la presentación del semen, aunque algunos autores como O. Azawi y M. Al-Mola [47] reportan tasas de fertilidad de $91,5 \%$ con semen fresco. Esto se debe a que el esperma se deposita directamente en el útero y evade por completo la complejidad del canal cervical. Sin embargo, la utilización de esta técnica por parte de los medianos y pequeños productores es menor en comparación a las otras, debido que requiere mayor inversión de recursos económicos y personal capacitado. A diferencia de las técnicas anteriores, autores como A., Allaoui et al. [29], M. C. Gündüz et al. [28], C. Ghalsasi et al. [34] reportan tasas de preñez ypartos cercanos al $83 \%$ con la utilización del protocolo de sincronización largo y monta natural.

Tabla 5. Técnicas de inseminación artificial vs protocolo de sincronización corto

\begin{tabular}{|c|c|c|c|c|c|c|c|c|c|c|c|c|c|}
\hline \multirow{4}{*}{ Autores } & \multicolumn{13}{|c|}{ PROTOCOLO DE SINCRONIZACIÓN CORTO } \\
\hline & \multicolumn{10}{|c|}{ Técnicas de inseminación artificial } & \multirow{2}{*}{\multicolumn{3}{|c|}{ MONTA NATURAL }} \\
\hline & \multicolumn{4}{|c|}{ IAC } & \multicolumn{3}{|c|}{ IATC } & \multicolumn{3}{|c|}{ IAL } & & & \\
\hline & SEMEN & $\mathrm{TF}$ & $\mathrm{TP}$ & $\begin{array}{c}\mathrm{T} \\
\text { PARTOS }\end{array}$ & $\mathrm{TF}$ & $\mathrm{TP}$ & $\begin{array}{c}\mathrm{T} \\
\text { PARTOS }\end{array}$ & $\mathrm{TF}$ & $\mathrm{TP}$ & $\begin{array}{c}\mathrm{T} \\
\text { PARTOS }\end{array}$ & $\mathrm{TF}$ & $\mathrm{TP}$ & $\begin{array}{c}\mathrm{T} \\
\text { PARTOS }\end{array}$ \\
\hline $\begin{array}{l}\text { R. Casali et al., } \\
\text { 2017(32) }\end{array}$ & Fresco & - & 36 & - & - & - & - & - & 50,2 & - & - & - & - \\
\hline $\begin{array}{l}\text { K. D. Antunes-Melo } \\
\text { et al., } 2015 \text { (38) }\end{array}$ & Congelado & - & - & - & - & 46,6 & - & - & - & - & - & - & - \\
\hline $\begin{array}{l}\text { FUKUI et al., } \\
1993(42)\end{array}$ & Fresco & - & - & - & - & - & - & - & 58 & - & - & - & - \\
\hline $\begin{array}{l}\text { B. Depaz et al., } \\
2011(45)\end{array}$ & Congelado & - & - & - & - & - & - & - & - & - & - & - & 69,4 \\
\hline
\end{tabular}

TF: tasa de fertilidad

TP: tasa de preñez

Fuente: elaboración propia.

Tabla 6. Técnicas de inseminación artificial vs celo natural

\begin{tabular}{|c|c|c|c|c|c|c|c|}
\hline \multirow{4}{*}{ Autores } & \multicolumn{7}{|c|}{ PROTOCOLO DE SINCRONIZACIÓN NATURAL } \\
\hline & & \multicolumn{6}{|c|}{ Técnicas de IA } \\
\hline & & \multicolumn{3}{|c|}{ IAC } & \multicolumn{3}{|c|}{ IAL } \\
\hline & SEMEN & TF & TP & T PARTOS & TF & TP & T PARTOS \\
\hline $\begin{array}{l}\text { L F Pfeifer et al., } \\
2012(23)\end{array}$ & Congelado & - & $71,1^{*}$ & - & - & 66,7 & - \\
\hline $\begin{array}{l}\text { J. Manes et al., } \\
\text { 2014(27) }\end{array}$ & Fresco & - & 55,4 & - & - & - & - \\
\hline
\end{tabular}

TF: tasa de fertilidad

TP: tasa de preñez

Fuente: elaboración propia. 


\section{Conclusiones}

Los principales factores que dificultan la preñez por IA en ovinos son: la edad de los animales, los parámetros morfométricos del cérvix, el tipo de técnica de iA y la presentación del semen.

La edad de las ovejas representa un factor decisivo en el éxito de la inseminación artificial. En los animales jóvenes se presentan las mayores dificultades, en razón a que el cérvix es corto, estrecho, y con un mayor número de anillos cervicales, circunstancia que dificulta el paso del catéter, en comparación de las hembras adultas las cuales presentan un cérvix largo, ancho y con un menor número de anillos cervicales.

Las formas de orificio externo cervical no parecen estar relacionadas con la dificultad en la ejecución de la ia. Tampoco se encontró relación de estas formas con los parámetros morfométricos del cérvix (longitud, diámetro y número de anillos), como tampoco con los parámetros reproductivos.

El diámetro de los anillos cervicales es un factor determinante en la implementación de IA, debido que el punto de menor diámetro en el cérvix determina la profundidad de penetración de catéter $y$, de igual manera, contribuye la eficiencia reproductiva cuando se utilizan las técnicas de la IA.

La utilización de semen congelado es un factor que limita la preñez por IA. Cuando se implementa IAV e IAC, esta limitación tal vez se debe a la incapacidad del semen y la larga trayectoria que debe recorrer hasta el útero.

\section{Referencias}

[1] Lonergan P. Review: Historical and futuristic developments in bovine semen technology. Animal. 2018;12(s1):s4-18. doi: 10.1017/S175173111 $800071 \mathrm{X}$.

[2] Muñoz DL. Acuerdo nacional de competitividad. Cadena productiva ovino-caprina nacional. Ministerio de Agricultura y Desarrollo Rial [Internet]. 2012;1-89. Disponible en: https://sioc.minagricultura.gov.co/OvinoCaprina/Normatividad/Nuevo $\% 20$ Acuerdo\%20Nacional\%20de\%20Competitividad\%202012.pdf

[3] Instituto Colombiano Agropecuario. Censo Pecuario Nacional - 2017. 2017 [Internet]. Disponible en: https://www.ica.gov.co/Areas/Pecuaria/Servicios/Epidemiologia-Veterinaria/Censos-2016/Censo-2017.aspx
[4] ICA. Censo-ovino-2017 [Internet]. Disponible en: https://www.ica.gov.co/Areas/Pecuaria/Servicios/ Epidemiologia-Veterinaria/Censos-2016/Censo-2017.aspx

[5] Kaabi M, Álvarez M, Anel E, Chamorro CA, Boixo JC, de Paz P, et al. Influence of breed and age on morphometry and depth of inseminating catheter penetration in the ewe cervix: A postmortem study. Theriogenology Theriogenology. 2006 nov.;66(8):1876-83. doi: 10.1016/j.theriogenology.2006.04.039

[6] Souza M, Nadal Da S, Dias Goncalves P NJ. Características morfométricas y penetrabilidad. Cienc Rural St Maria. 1994;24(3):591-5.

[7] Cruz Júnior CA, Mcmanus C, Jivago JLPR, Bernardi M, Lucci CM. Anatomical and histological characterization of the cervix in Santa Inês hair ewes. Anim Reprod [Internet]. 2014;11(1):49-55. Disponible en: http://www.cbra.org.br/pages/publicacoes/ animalreproduction/issues/download/v11n1/pag49-55(AR594).pdf

[8] Franco MC, dos Santos JF, Maciel TA, Neto PJD, Oliveira D. Morphology of the cervix of santa ines adult sheep in luteal and folicular phases | Morfologia da cérvice de ovelhas santa inês adultas nas fases luteínica e folicular. Cienc Anim Bras. 2014;15(4): 495-501.

[9] Gultiken N, Gultiken ME, Anadol E, Kabak M, Findik M. Morphometric study of the cervical canal in Karayaka ewe. J Anim Vet Adv. 2009;8(11):2247-50.

[10] Breda JC, Sotomaior C. Dados de alguns parâmetros anatômicos do aparelho reprodutivo de ovelhas da some anatomical parameters of the reproductive tract from sheep of the Hampshire Down and Crossbre Hampshire Down-Ile de France. 2007;5(3): 237-242.

[11] Naqvi SMK, Pandey GK, Gautam KK, Joshi A, Geethalakshmi V, Mittal JP. Evaluation of gross anatomical features of cervix of tropical sheep using cervical silicone moulds. Anim Reprod Sci. 2005 Feb;85(34):337-44. doi: 10.1016/j.anireprosci.2003.10.007

[12] Eppleston J, Salamon S, Moore NW, Evans G. The depth of cervical insemination and site of intrauterine insemination and their relationship to the fertility of frozen-thawed ram semen. Anim Reprod Sci. 1994;36(3-4):211-25.

[13] Leethongdee S. Development of Trans-Cervical Artificial Insemination in Sheep With Special Reference To Anatomy of Cervix. Suranaree J Sci Technol. 2016;17(1):57-69.

[14] Halbert GW, Dobson H, Walton JS, Buckrell BC. The structure of the cervical canal of the ewe. Theriogenology 2017;33(5):977-92. doi: https://doi. org/10.1016/0093-691X(90)90060-7 
[15] Dun RB. The Cervix of the Ewe-Its Importance in Artificial Insemination of Sheep. Aust Vet J. 1955;13. doi: https://doi.org/10.1111/j.1751-0813.1955. tb05513.x

[16] Moura DS, Lourenço TT, Moscardini MM, Scott C, Fonseca PO, Souza FF. Aspectos morfológicos da cérvice de ovelhas. Pesqui Vet Bras. 2011;31(suppl. 1):33-8.

[17] Richardson L, Hanrahan JP, Donovan A, MartíJI, Fair S, Evans ACO, et al. Effect of site of deposition on the fertility of sheep inseminated with frozen-thawed semen. Anim Reprod Sci. 2012;131(3):160-4. doi: 10.1016/j.anireprosci.2012.03.006.

[18] Kershaw CM, Khalid M, McGowan MR, Ingram K, Leethongdee S, Wax G, et al. The anatomy of the sheep cervix and its influence on the transcervical passage of an inseminating pipette into the uterine lumen. Theriogenology. 2005;64(5):1225-35.

[19] Álvarez, M.; Kaabi, M.; Boixo, J.C.; Anel, E.; Chamorro, CA;Martínez, S. García C; Anel L. Valoración morfométrica del canal cervical de la oveja mediante imágenes de resonancia magnética. Itea Prod Anim. 2001; Extra:3-5.

[20] Candappa I, Bartlewski P. A review of advances in artificial insemination (AI) and embryo transfer (ET) in sheep, with the special reference to hormonal induction of cervical dilation and its. Open Reprod Sci J 2011;3:162-75. doi: 10.2174/1874255601103010162

[21] Fang Y, Zhong R, Zhang X, Zhang J, Zhou D. Boar seminal plasma inhibits cryo-capacitation of frozen-thawed ram sperm and improves fertility following intracervical insemination. Theriogenology 2018;105:84-9. doi: https://doi.org/10.1016/j.theriogenology.2017.09.011

[22] Barbas JP, Horta AEM, Marques CC, Baptista MC, Mascarenhas RD, Martins DO, et al. The fertility increase after misoprostol administration is differently expressed when sheep are inseminated with chilled or frozen-thawed semen. Small Rumin Res. 2013;113(2-3):398-401.

[23] Pfeifer LFM, Rabassa VR, Neto JW da S, Meneghello L de C, Castilho EM, Severo NC, et al. Efeito da aplicação de flunixin meglumine sobre a taxa de gestação de ovelhas inseminadas via transcervical. Acta Sci Vet. 2010;38(3):307-10.

[24] Anel L, Kaabi M, Abroug B, Alvarez M, Anel E, Boixo JC, et al. Factors influencing the success of vaginal and laparoscopic artificial insemination in churra ewes: A field assay. Theriogenology. 2005;63(4):1235-47.

[25] Masoudi R, Zare Shahneh A, Towhidi A, Kohram H, Akbarisharif A, Sharafi M. Fertility response of artificial insemination methods in sheep with fresh and frozen-thawed semen. Cryobiology. 2017;74:77-80.
[26] Horta AEM, Barbas JP, Marques CC, Baptista MC, Vasques MI, Pereira RM, et al. Improvement of fertility in artificially inseminated ewes following vaginal treatment with misoprostol plus terbutaline sulphate. Reprod Domest Anim. 2010;45(6):412-6.

[27] Manes J, Hozbor F, Alberio R, Ungerfeld R. Intravaginal placebo sponges affect negatively the conception rate in sheep. Small Rumin Res. 2014;120(1):108 11. doi: http://dx.doi.org/10.1016/j.smallrumres.2014.05.006

[28] Gündüz MC, Turna Ö, Cirit Ü, Uçmak M, Tek Ç, Sabuncu A, et al. Lambing rates and litter size following carazolol administration prior to insemination in Kivircik ewes. Anim Reprod Sci. 2010;118(1):32-6.

[29] Allaoui A, Tlidjane M, Safsaf B, Laghrour W. Comparative Study between Ovine Artificial Insemination and Free Mating in Ouled Djellal Breed. APCBEE Procedia 2014;8(Caas 2013):254-9. doi: https://doi.org/10.1016/j.apcbee.2014.03.036

[30] Cardoso E, Da Cruz, JF, Ferraz RC. N.; Teixeira-Neto MR, Dos Santos RS Avaliação econômica de diferentes técnicas de inseminação artificial em ovinos da raça Santa Inês 1. Rev Bras Ciências Agrárias. 2009;4(2):217-22.

[31] Álvarez M, Chamorro CA, Kaabi M, Anel-López L, Boixo JC, Anel E, et al. Design and «in vivo» evaluation of two adapted catheters for intrauterine transcervical insemination in sheep. Anim Reprod Sci. 2012;131(3-4):153-9. doi: http://dx.doi. org/10.1016/j.anireprosci.2012.03.001

[32] Casali R, Pinczak A, Cuadro F, Guillen-Muñoz JM, Mezzalira A, Menchaca A. Semen deposition by cervical, transcervical and intrauterine route for fixed-time artificial insemination (FTAI) in the ewe. Theriogenology. 2017; 103:30-5.

[33] Andersen VK, Aamdal J, Fougner JA. Intrauterine and deep cervical insemination with frozen semen in sheep. Reprod Domest Anim. 1973;8(3):113-8.

[34] Ghalsasi PM, Nimbkar C. Evaluation of laparoscopic intrauterine insemination in ewes. Small Rumin Res. 1996;23(1):69-73.

[35] Fierro S, Olivera-Muzante J. Long interval prostaglandin as an alternative to progesterone-eCG based protocols for timed AI in sheep. Anim Reprod Sci. 2017; 180:78-84. doi: http://dx.doi.org/10.1016/j. anireprosci.2017.03.004

[36] Rashidi M, Cedden F. Trans-Cervical Artificial Insemination in Ewes During Out of Breeding Season. Maced J Anim Sci. 2013;3(2):143-6.

[37] Naim, P. Cueto, M. Gibbons A. Inseminación Artificial a Tiempo Fijo (I.A.T.F). Arch Zootec [Internet] 2009;46(223):203,206. Disponible en: https://inta. gob.ar/sites/default/files/script-tmp-inta_ganaderia46_inseminacion_ovina.pdf 
[38] Antunes-Melo KD, Oliveira VS, Santos ADF, Oliveira CA, Mendonça LM, Goveia JSS, et al. Use of reduced doses of eCG applied by different routes in the TAI program in Santa Ines sheep. Semin Ciências Agrárias. 2015;36(3):1347-54. doi: 10.5433/1679-0359.2015v36n3p1347

[39] Kohram H, Seifi-Jamadi, Masoudi R, Sharafi M, Moein Aledavood SD, Sadeghpanah A. Effects of estradiol and oxytocin injection on the efficiency of artificial insemination in Iranian Zel ewes during the breeding season. Arch Razi Inst. 2017;72(1):3341. doi: DOI: http://dx.doi.org/10.22034/ari.2016. 107489

[40] Masoudi R, Shahneh AZ, Towhidi A, Kohram H, Akbarisharif A, Sharafi M, et al. Cervical dilation and improvement of reproductive performance in fat-tailed ewes via cervical dilator treatments. Asian Pacific J Reprod. 2017;6(2):93-6. doi: doi: 10.12980/ apjr.6.20170209

[41] Avendeño-Reyes L, Alvarez-Valenzuela FD, Molina-Ramírez L, Rangel-Santos R, Correa-Calderón A, Rodríguez-García J, et al. Reproduction performance of Pelibuey ewes in response to estrus synchronization and artificial insemination in Northwestern Mexico. Journal of Animal and Veterinary Advances [Internet]. 2007;6(7): Disponible en: 807-12. http://medwelljournals.com/abstract/?doi=javaa.2007.807.812
[42] Fukui Y, Fujii M, Tashiro Y. Insemination Doses of Frozen-Thawed Semen in Seasonally Anestrous Ewes Treated with Two Different Progesterone-Impregnated Intravaginal Devices. J Reprod Dev Reprod Dev [Internet]. 1993;39(39):269-73. Disponible en: https://www.jstage.jst.go.jp/article/ jrd1992/39/4/39_4_269/_pdf/-char/en

[43] Fukui Y, Kohno H, Togari T, Hiwasa M, Okabe K. Fertility after artificial insemination using a soybean-based semen extender in sheep. J Reprod Dev. 2008;54(4):286-9.

[44] Mellisho E, Pinazo R, Chauca L, Cabrera P, Rivas V. Inseminación intrauterina vía laparoscópica de ovejas black belly con semen congelado. Rev Inv Vet. 2006;17(2):131-6.

[45. Depaz B, Álvarez Y, Mellisho E. Efecto del tratamiento corto de progesterona en la fertilidad de ovinos pelibuey inseminado vía laparoscopia con semen congelado. SPERMOVA. 2011;1(jul.):123-4.

[46] Hidalgo G, Rodríguez-Márquez J, Chango R, Mavarez M, Morales R, Rodríguez M, et al. Inseminación intrauterina por laparoscopia en ovejas mestizas West African utilizando semen Dorper congelado en pajuelas y pellets. Rev Cient Vet. 2015;25(5): 395-401.

[47] Azawi OI, Al-Mola MK. A study on the effect of $\mathrm{GnRH}$ administration on the ovarian response and laparoscopic intrauterine insemination of Awassi ewes treated with eCG to induce superovulation. Trop Anim Health Prod. 2011;43(7):1351-5. doi: 10.1007/s11250-011-9856-7. 